Journal of Breath Research

\title{
Quantitative analysis of volatile metabolites released in vitro by bacteria of the genus Stenotrophomonas for identification of breath biomarkers of respiratory infection in cystic fibrosis.
}

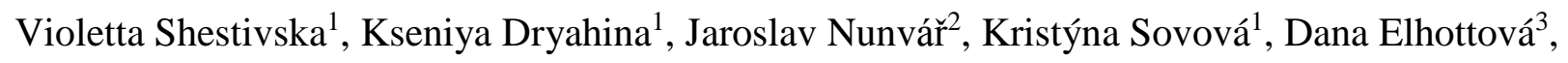
Alexandr Nemec ${ }^{4}$, David Smith ${ }^{5}$ and Patrik Španěl ${ }^{1 *}$

${ }^{1}$ J. Heyrovsky Institute of Physical Chemistry, Academy of Sciences of the Czech Republic, Dolejškova 3, 18223 Prague 8, Czech Republic

2 Department of Medical Microbiology 2nd Faculty of Medicine, Charles University in Prague and Motol University Hospital, V Uvalu 84, 15006, Prague 5, Czech Republic

${ }^{3}$ Institute of Soil Biology, Biology Centre of the Academy of Sciences of the Czech Republic, 37005

České Budějovice, Czech Republic

${ }^{4}$ Laboratory of Bacterial Genetics, National Institute of Public Health, Šrobárova 48, 10042 Prague,

Czech Republic

${ }^{5}$ Institute for Science and Technology in Medicine, School of Medicine, University of Keele, Thronburrow Drive, ST4 7QB, Stoke on Trent, UK.

*Correspondence to spanel@jh-inst.cas.cz J. Heyrovsky Institute of Physical Chemistry, Academy of Sciences of the Czech Republic, Dolejškova 3, 18223 Prague 8, Czech Republic.

Keywords: volatile metabolites, Stenotrophomonas, cystic fibrosis, selected ion flow tube mass spectrometry (SIFT-MS), gas chromatography mass spectrometry (GC-MS) 


\begin{abstract}
The aim of the present study was to characterise the volatile metabolites produced by genotypically diverse strains of the Stenotrophomonas genus in order to evaluate their potential as biomarkers of lung infection by non-invasive breath analysis. Volatile organic compounds (VOCs) emitted from 15 clinical and five environmental strains belonging to different genogroups of Stenotrophomonas maltophilia (n=18) and Stenotrophomonas rhizophila $(\mathrm{n}=2)$ cultured in Mueller-Hinton Broth (MHB) liquid media were analysed by gas chromatography mass spectrometry (GC-MS) and selected ion flow tube mass spectrometry (SIFTMS). Several VOCs were detected in high concentration, including ammonia, propanol, dimethyl disulphide, and dimethyl trisulphide. The GC-MS measurements showed that all 15 clinical strains produced similar headspace VOCs compositions and SIFT-MS quantification showed that the rates of production of the VOCs by the genotypically distinct strains were very similar. All in vitro cultures of both the Stenotrophomonas species were characterised by efficient production of two isomers of methyl butanol, which can be described by known biochemical pathways and which is absent in other pathogens including Pseudomonas Aeruginosa . These in-vitro data indicate that methyl butanol isomers may be exhaled breath biomarkers of $S$. maltophilia lung infection in patients with cystic fibrosis.
\end{abstract}




\section{Introduction}

The genus Stenotrophomonas encompasses Gram-negative, strictly aerobic bacteria which are ubiquitous in aqueous environments, soil and plants [1]. These organisms have also been used for biotechnology applications [2]. Although they are generally non-pathogenic to healthy people, they may cause serious infections in patients with severe underlying disease or impaired immunity. Risk factors associated with Stenotrophomonas infection include HIV infection, malignancy, cystic fibrosis, neutropenia, the presence of mechanical ventilation or central venous catheters, recent surgery, trauma, and therapy using broad-spectrum antibiotics. The number of Stenotrophomonas infections in humans has dramatically increased in recent years, especially in patients with cystic fibrosis (CF) [3]. The lack of mucociliary clearance in the airways of CF patients leads to the build-up of immovable mucus in the lungs and airways of CF patients provides a substrate for colonization by different bacteria, notably Pseudomonas aeruginosa, [4], Staphylococcus aureus and Streptococcus pneumoniae [5], Stenotrophomonas spp. with which the present paper is largely concerned, and the fungus Aspergillus fumigatus [6]. Early diagnosis and antibiotic treatment of such infections can improve the prognosis and extend the patients' life [7]. Some details of the genetic aspects of the genus Stenotrophomonas are given in Appendix 1 and the biochemical routes to the production of volatile organic compounds (VOCs) by this bacterium are given in Appendix 2.

Breath analysis holds promise as a non-invasive diagnostic of lower respiratory tract infections, obviating the routinely-used invasive procedures like bronchoscopy or alveolar lavage [8]. Our recent SIFT-MS studies of VOCs released by in vitro cultures of the bacteria P. aeruginosa, S. aureus, S. pneumoniae, and the fungus $A$. fumigatus [6], suggest that these VOCs may appear in exhaled breath of persons infected with these pathogens. The non-invasive breath analysis approach to diagnosis would be 
of great value, especially in children with CF where early detection of respiratory pathogens increases the chance of eradication and improves prognosis [9].

Analyses of volatile compounds (VOCs) emitted from Stenotrophomonas bacterial cultures in vitro is a natural extension of our on-going search for volatile biomarkers of lung and airways infections by breath analysis. Selected ion flow tube mass spectrometry (SIFT-MS) is being successfully used for on-line, real time quantification of trace gases in human breath $[10,11]$ and has great potential as a tool for non-invasive physiological monitoring of CF patients [4, 9]. A notable recent advance is the identification of HCN as a biomarker of $P$. aeruginosa in CF patients; studies based on SIFT-MS have shown that both HCN [4, 9] and methylthiocyanate [12] are specific volatile biomarkers of this pathogen. SIFT-MS has been recently exploited to quantify a range of volatile metabolites released by various invitro cultures of P. aeruginosa [13] and other respiratory pathogens [5, 6]. Similarly, gas chromatographymass spectrometry (GC-MS) is a well-established technique for untargeted analyses of volatile compounds in complex matrices and has been exploited to identify many of the characteristic VOCs released by bacterial cultures $[14,15]$.

The present study aimed to assess the VOCs released by in vitro cultures of Stenotrophomonas spp. in order to identify potential biomarkers of infection of the airways by these bacteria in CF patients via breath analysis. This work, first presented at the Breath Analysis 2014 meeting in Torun, Poland, has been conducted using the powerful combination of GC-MS and SIFT-MS by which VOCs released by a genotypically characterized bacterial collection encompassing 18 strains of S. maltophilia isolated from clinical $(n=15)$ and environmental $(n=3)$ specimens and two strains of $S$. rhizophila of environmental origin have been investigated. 


\section{Materials and methods}

\section{Origin and genetic diversity of the bacterial strains}

The study set encompassed 20 Stenotrophomonas strains, including 15 S. maltophilia strains isolated from clinical specimens and five environmental strains (three S. maltophilia and two $S$. rhizophila) from natural environments (Table 1). Nine of the 15 clinical strains were recovered from the respiratory specimens of CF patients at the Cystic Fibrosis Centre in Prague. The taxonomic affiliation of these 20 strains were determined previously [16]. The previous work using multi-locus sequence typing (MLST) has delineated nine different genogroups (GGs) [17]. The clinical strains included in the present study belong to at least four of these genogroups (GGs) with GG6 containing most of them. All five environmental strains belong to either the strictly environmental species S. rhizophila (previous GG8) or GG9 [18-20].

Table 1. The 20 Stenotrophomonas strains studied

\begin{tabular}{|c|c|c|c|}
\hline Strain $^{*}$ & Species (genogroup) & Specimen & Year of isolation \\
\hline${\text { CNCTC } 5821^{\top}}^{\top}$ & S. maltophilia (GG6) & Orypharynx & Before 1964 \\
\hline ANC 4262 (= Cf1) & S. maltophilia (GG6) & CF sputum & 2004 \\
\hline ANC $4263(=\mathrm{Cf} 2)$ & S. maltophilia (GG6) & CF throat swab & 2005 \\
\hline ANC 4264 (= Cf3) & S. maltophilia (GG6) & CF sputum & 2005 \\
\hline ANC 4265 (= Cf4) & S. maltophilia (GGnew) & CF sputum & 2005 \\
\hline ANC 4266 (= Cf5) & S. maltophilia & CF sputum & 2005 \\
\hline ANC 4267 (= Cf6) & S. maltophilia (GG6) & CF sputum & 2006 \\
\hline ANC $4268(=\mathrm{Cf} 7)$ & S. maltophilia (GG6) & CF sputum & 2006 \\
\hline ANC 4269 (= Cf8) & S. maltophilia (GG1) & CF sputum & 2007 \\
\hline ANC 4331 (= Cf9) & S. maltophilia (GG6) & CF sputum & 1989 \\
\hline ANC $4332(=\mathrm{Cli} 4)$ & S. maltophilia (GGnew) & Blood culture & 1994 \\
\hline ANC 4333 (= Cli5) & S. maltophilia (GG4) & Endotracheal tube & 1994 \\
\hline ANC 4336 (= Cli2) & S. maltophilia & Venous catheter & 1993 \\
\hline ANC 4338 (= Cli7) & S. maltophilia (GG6) & Wound & 1997 \\
\hline ANC 4339 (= Cli8) & S. maltophilia (GGnew) & Blood & 1997 \\
\hline ANC 4341 (= Env1 ) & S. maltophilia (GG9) & Meadow & 2007 \\
\hline ANC 4342 (= Env2) & S. maltophilia (GG9) & Meadow & 2007 \\
\hline ANC 4343 (= Env11) & S. maltophilia (GG9) & Cave & 2010 \\
\hline ANC 4344 (= Env13) & S. rhizophila (GG8) & Cave & 2010 \\
\hline ANC 4345 (= Env14) & S. rhizophila (GG8) & Cave & 2010 \\
\hline
\end{tabular}


* Strain codes used by Nurvar et al. [16] are in parentheses.

\# Assignation to genogroup based on partial gyrB gene sequence analysis [17].

\section{Culturing and quantification of bacteria}

Liquid (submersion) cultures of each strain were prepared in $7 \mathrm{~mL}$ of Mueller-Hinton Broth (MHB) (Oxoid Ltd, UK) in $100 \mathrm{~mL}$ hermetically sealed flasks [12]. These cultures were inoculated with $\sim 50 \mu \mathrm{L}$ of cell suspension of standardized turbidity $\left(\sim 10^{8}\right.$ colony forming units [CFU] $\left.\mathrm{mL}^{-1}\right)$ prepared in saline from an overnight agar culture, and these were cultured with continuous stirring in a thermostatically controlled water bath for $20 \mathrm{~h}$. The cultures of the 15 clinical strains were grown at $37^{\circ} \mathrm{C}$ whereas the cultivation temperature for the five environmental strains had to be lowered to $30^{\circ} \mathrm{C}$ because of insufficient growth at $37^{\circ} \mathrm{C}$. As a control, sterile liquid MHB medium cultured under the same conditions was included in all experiments. All strains and controls were processed in duplicate within each experiment. Bacterial growth was assessed both by CFU counting and by optical density measurement. The CFU mL ${ }^{-1}$ of all strains cultured for $20 \mathrm{~h}$ ranged from $0.9 \times 10^{10}$ to $1.9 \times 10^{10}$.

\section{SPME headspace sampling protocol and GC-MS analysis.}

VOCs were extracted from the headspace of the bacterial cultures using solid phase microextraction (SPME) onto carboxen/polydimethylsiloxane (CAR/PDMS)-coated fibres (Supelco, Bellefonte, PA, USA) for $30 \mathrm{~min}$ at a temperature $37^{\circ} \mathrm{C}$. Immediately following the extraction period, the SPME fibres were directly inserted into the injector of the GC-MS instrument (FOCUS GC with SSL, ITQ 700 ion trap mass spectrometer using electron ionisation) held at $210^{\circ} \mathrm{C}$. The GC conditions were the following: splitless injection, He carrier gas at $1 \mathrm{~mL} / \mathrm{min}$, GC oven temperature program $38^{\circ} \mathrm{C}$ (hold 13 min) $3^{\circ} \mathrm{C} / \mathrm{min}$ ramp up to $100^{\circ} \mathrm{C}$ (hold $5 \mathrm{~min}$ ), $4^{\circ} \mathrm{C} / \mathrm{min}$ to $150,20^{\circ} \mathrm{C} / \mathrm{min}$ to $210^{\circ} \mathrm{C}$ and a final hold $3 \mathrm{~min}$ (total run time 58 min). A GC-MS capillary column TG-624 (fused 100\% Cyanopropylphenyl Polisiloxane, $30 \mathrm{~m} \times 0.25 \mathrm{~mm}$ ID x $1.0 \mathrm{um}$ film) was used. Electron ionisation at $70 \mathrm{eV}$ generated ions that 
were analysed by the ion trap operating in the scan mode $(\mathrm{m} / \mathrm{z} 15-400$, scan rate $1 \mathrm{scan} / \mathrm{s})$. Peak identification was based on mass spectral interpretation and comparisons with the NIST 2.0 library [21]. Kovats retention indices were obtained by analysing standard mixtures of different $\mathrm{C}_{5}-\mathrm{C}_{10}$ hydrocarbons. Whilst the SPME/GC-MS technique is a suitable method for identification of unknown molecules in complex matrices, quantification of compounds is time consuming and not precise. SIFT-MS is able to accurately quantify specific VOCs in complex mixtures in real time and so the combination of SPME/GCMS and SIFT-MS is powerful for the analysis of complex mixtures.

\section{SIFT-MS analysis}

The SIFT-MS analytical technique has been described in detail previously [10, 11, 22] and the particular aspects of its use to analyse in vitro bacterial cultures are detailed in the previous papers by Shestivska et al $[12,13]$. Thus, the headspace of the bacterial cultures was analysed using judiciously chosen reagent ions $\left(\mathrm{H}_{3} \mathrm{O}^{+}, \mathrm{NO}^{+}\right.$or $\left.\mathrm{O}_{2}^{+}\right)$to produce analyte ions that identify the neutral trace compounds present in the headspace [10]; the the count rates of these analyte ions provide accurate absolute quantification of the precursor neutral trace compounds down to concentrations as low as parts-per-billion by volume, ppbv [23-25]. A list of compounds quantified by SIFT-MS, together with the reagent ions and the analyte ions used for their identification and quantification, is given in Table 2. Some of the compounds identified by GC-MS analyses were specifically targeted for SIFT-MS analysis. The data

obtained were assessed by principal component analysis (PCA) using the Statistica software (StatSoft Czech Republic s.r.o.), as explained and discussed later. 
Table 2. Reagent and product ions used for SIFT-MS quantification of volatile metabolites in the headspace of cultures of the 20 Stenotrophomonas strains. Note that SIFT-MS quantifications correspond to the sum of concentrations of all isomers of the compounds indicated.

\begin{tabular}{rlll}
\hline SIFT-MS & $\begin{array}{l}\mathrm{MW} \\
\mathrm{g} / \mathrm{mol}\end{array}$ & $\begin{array}{l}\text { reagent } \\
\text { ion }\end{array}$ & $\begin{array}{l}\text { Analyte (product) ions } \\
\mathrm{m} / \mathrm{z}\end{array}$ \\
\hline Methanol & 32 & $\mathrm{H}_{3} \mathrm{O}^{+}$ & $33,51,69$ \\
Propanol & 60 & $\mathrm{H}_{3} \mathrm{O}^{+}$ & 43 \\
Butanol & 74 & $\mathrm{H}_{3} \mathrm{O}^{+}$ & $57,75,93$ \\
Methyl butanol & 88 & $\mathrm{H}_{3} \mathrm{O}^{+}$ & $71,89,107$ \\
Hydrogen sulphide & 34 & $\mathrm{H}_{3} \mathrm{O}^{+}$ & 35,53 \\
Methanethiol & 48 & $\mathrm{H}_{3} \mathrm{O}^{+}$ & $49,67,85$ \\
Dimethyl sulphide & 62 & $\mathrm{O}_{2}{ }^{+}$ & 62,80 \\
Dimethyl disulphide & 94 & $\mathrm{NO}^{+}$ & 94 \\
Butanone & 72 & $\mathrm{NO}^{+}$ & 102 \\
Ammonia & 17 & $\mathrm{O}_{2}{ }^{+}$ & 17,35 \\
Methyl benzoate & 136 & $\mathrm{H}_{3} \mathrm{O}^{+}$ & 137 \\
Pentane & 72 & $\mathrm{O}_{2}{ }^{+}$ & 42,72 \\
Butyric acid & 88 & $\mathrm{NO}^{+}$ & 71,118 \\
\hline & & &
\end{tabular}

\section{Results and discussion}

Sample chromatograms obtained by headspace SPME/GC-MS analyses of cultivated sterile medium and of identical media cultivated with clinical and environmental S. maltophilia strains are shown in Fig. 1. The GC-MS data show that the qualitative composition of the produced VOCs (see Table 3) is very similar for all 20 Stenotrophomonas strains studied. 


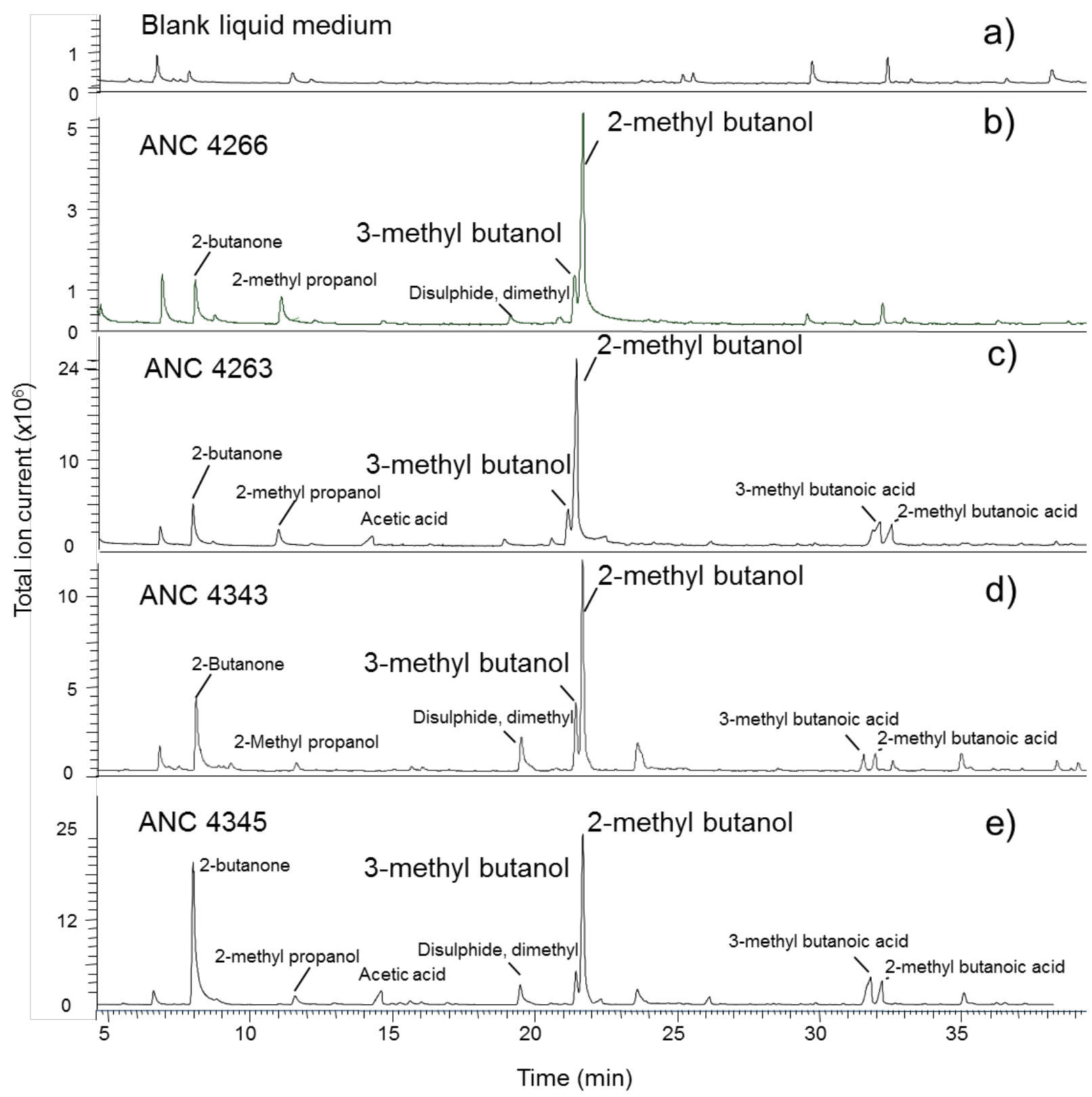

Fig. 1. GC-MS analyses of VOCs extracted using SPME from the headspace of a) blank sample (liquid MHB medium), b) identical medium cultivated with the clinical S. maltophilia strain ANC 4266 c) clinical S. maltophilia strain ANC 4263 (GG6), d) environmental S. maltophilia strain ANC 4343(GG9), e) environmental S. rhizophila strain ANC 4345 (GG8).

The SIFT-MS analyses provided absolute concentrations of 13 VOCs given in Fig. 2, but were unable to distinguish the two methyl butanol isomers (both with a molecular weight of 88). SIFT-MS indicated presence of relatively high concentrations of ammonia (500 to 2000 ppbv), which is commonly produced by bacteria, including oral cavity bacteria, and so it cannot be used as a reliable volatile 
biomarker of lung infection. However, its elevation in the breath of patients may be a useful co-biomarker in combination with other specific volatiles such as methyl butanol (see below).

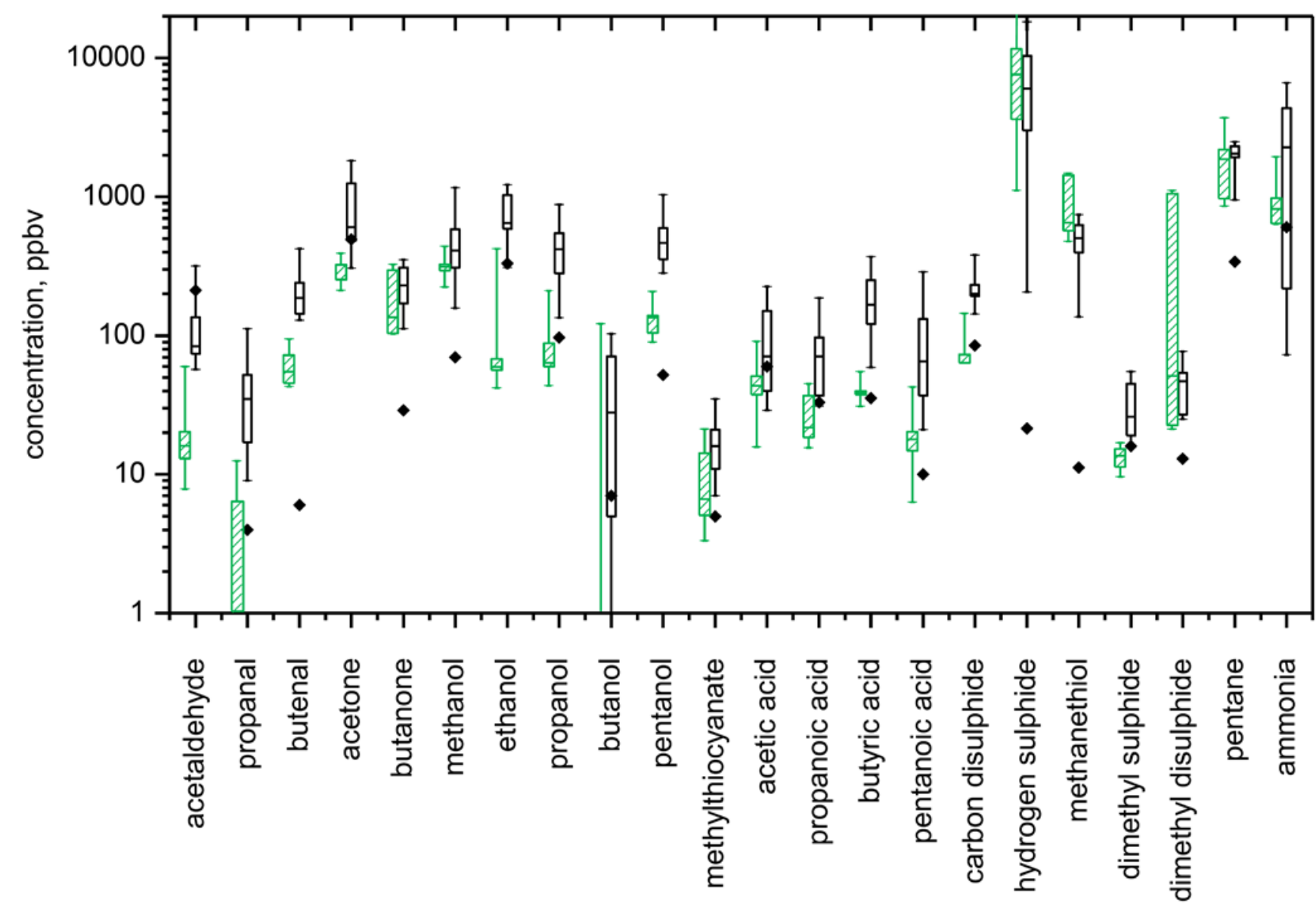

Fig. 2. SIFT-MS quantifications (in ppbv) of volatile metabolites detected in the headspace of cultures of the 20 Stenotrophomonas strains indicated by box and whiskers plots; dashed green for environmental and open black for clinical strains (whiskers indicate minimum and maximum range, boxes indicate $25^{\text {th }}$ and $75^{\text {th }}$ percentile and the lines indicate medians). The diamond data points show median concentrations in the headspace of cultured control medium.

Table 3 shows the VOCs identified by SPME/GC-MS in the culture headspace of the 20 Stenotrophomonas strains. Fourteen of these VOCs were seen in all the strains, including acetone, 2- 
butanone, 2-propanol, dimethyl disulphide, 2- and 3-methyl butanol. These VOCs can also be released by other bacterial cells, but in different concentration, as previously reported [5, 6, 12, 13].

Table 3. Volatile metabolites detected by SPME/GC-MS the headspace of cultures of the 20 Stenotrophomonas strains. Peak areas corresponding to the headspace concentrations of selected VOCs are indicated as percentages of observed maximum as: $\downarrow<25 \%$, o $=25-75 \%$ and $\bullet>75 \%$.

\begin{tabular}{lrrrcc}
\hline Compound & $\begin{array}{r}\text { Retention } \\
\text { time (m) }\end{array}$ & $\begin{array}{r}\text { Retention } \\
\text { index }\end{array}$ & Medium only & Clinical & Environmental \\
\hline 1-propanol & 7.03 & 616 & $\downarrow$ & $\circ$ & $\circ$ \\
2-propanol & 4.44 & 547 & $\circ$ & $\circ$ & $\circ$ \\
2-butanol & 9.38 & 650 & $\downarrow$ & $\circ$ & $\bullet$ \\
2-methyl-1-propanol & 12.23 & & $\downarrow$ & $\bullet$ & $\bullet$ \\
1-pentanol & 25.00 & 833 & $\downarrow$ & $\circ$ & $\bullet$ \\
3-methyl butanol & 22.48 & 800 & $\downarrow$ & $\bullet$ & $\bullet$ \\
2-methyl butanol & 22.76 & 804 & $\downarrow$ & $\bullet$ & $\bullet$ \\
dimethyl disulphide & 20.42 & 779 & $\downarrow$ & $\circ$ & $\bullet$ \\
dimethyl trisulphide & 38.04 & & $\downarrow$ & $\circ$ & $\circ$ \\
methyl thiolacetate & 16.40 & 737 & $\downarrow$ & $\circ$ & $\circ$ \\
2-butanone & 8.53 & 638 & $\circ$ & $\bullet$ & $\bullet$ \\
2-pentanone & 16.80 & 741 & $\downarrow$ & $\circ$ & $\circ$ \\
2-heptanone & 32.48 & 934 & $\downarrow$ & $\circ$ & $\circ$ \\
butyric acid & 29.04 & 885 & $\downarrow$ & $\circ$ & $\circ$ \\
\hline
\end{tabular}

Several organosulphur compounds were identified in the headspace of both the clinical and environmental Stenotrophomonas strains. Some of these compounds are commonly generated by bacteria, including those often present in the healthy human oral cavity responsible for oral malodour (hydrogen sulphide, methanethiol) [26-28]. However, one sulphur compound, dimethyl trisulphide, which was identified by SPME/GC-MS in the headspace of all 20 strains (see Table 2), is not usually released by non-Stenotrophomonas bacteria $[4,5]$ and, thus, may be considered as a potential volatile biomarker in exhaled breath specific to Stenotrophomonas infection. 
Several aldehydes, including methyl butanal, were observed in the headspace of the blank MHB liquid media and their concentrations decreased in the headspace of bacterial cultures; thus, they are not included in Table 2 and Table 3. This removal of aldehydes from culture media by bacterial and some mammalian cells has been observed in previous studies [29-32].

\section{Presence of methyl butanol isomers in the headspace of Stenotrophomonas cultures}

The SPME/GC-MS data show similar qualitative VOCs composition for all 20 strains. As examples of positive compound identification, total ion current chromatograms obtained for the sterile MHB liquid medium and four selected Stenotrophomonas strains are shown above in Fig.1. An important observation is that all the strains produced high amounts of two isomers of methyl butanol (Fig.1) (retention times $20.72 \mathrm{~min}$ for 3-methyl-1-butanol and $21.04 \mathrm{~min}$ for 2-methyl-1-butanol), so we have given special attention to these compounds. The elution times and the ionisation fragmentation patterns of these two compounds agreed with those of reference standard sample of these isomers and also with those included in the NIST ion mass spectral library NIST 2.0 [21]. Methyl butanol was observed in all the 20 strains (ranges of ion signals: $1.8 \times 10^{5}$ to $2.5 \times 10^{7}$ for 3-methyl-1-butanol and $3.2 \times 10^{7}$ to $2 \times 10^{8}$ for 2-methyl-1-butanol), the ion signal level of 3-methyl-1-butanol being typically 20\% of that for 2-methyl1-butanol, as indicated by the slope of the plot in Fig. 3. 


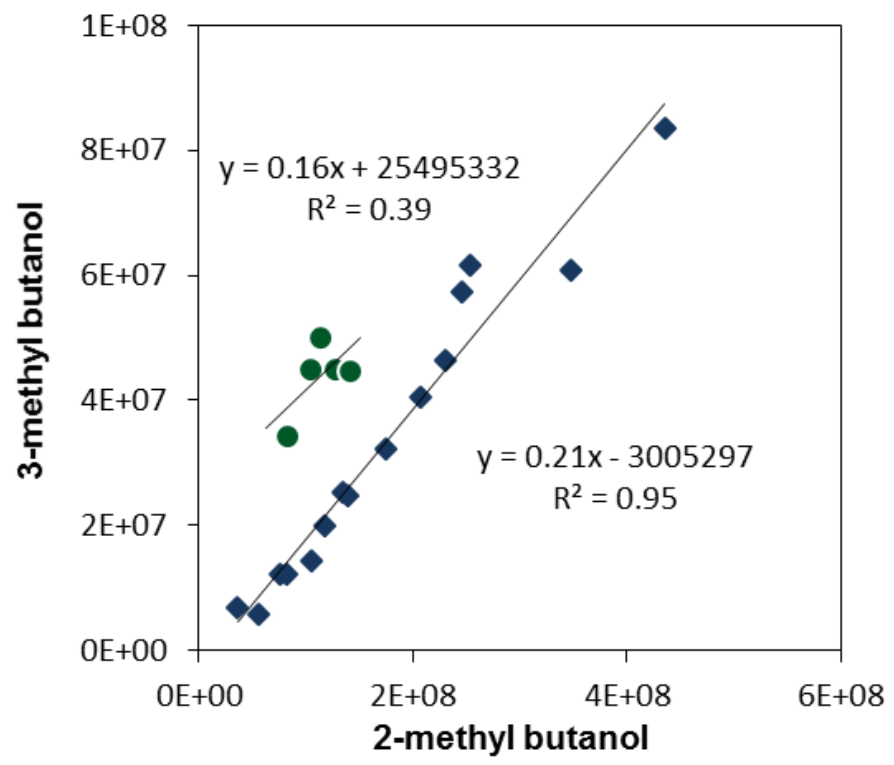

Fig. 3. GC-MS peak area correlation between 2-methyl butanol and 3-methyl butanol detected in the headspace of 20 Stenotrophomonas strains cultured in liquid MHB medium. The diamonds indicate the 15 clinical strains and the circles indicate the 5 environmental strains. Note the different correlations ( $\mathrm{R}^{2}$ values) for the two types of strain (see the text).

Fig. 3 also indicates the close correlation (more than 80\%) between the GC-MS signal peak areas for 2- and 3- methyl butanol generated by the 15 clinical Stenotrophomonas strains. The analogous correlation for the five environmental strains is weaker $\left(\mathrm{R}^{2}=0.39\right)$. This can be related to different metabolisms of those two groups of bacteria, but given the small number of samples this weaker correlation should not be over interpreted. Note that SIFT-MS quantification provides the total concentration of these two butanol isomers and their pentanol isomers; but, according to these GC-MS data, pentanol was present in the headspace of these bacteria strains in much smaller amounts (range of ion signals $<2 \times 10^{5}$ ) compared to the methyl butanol isomers (see above). 


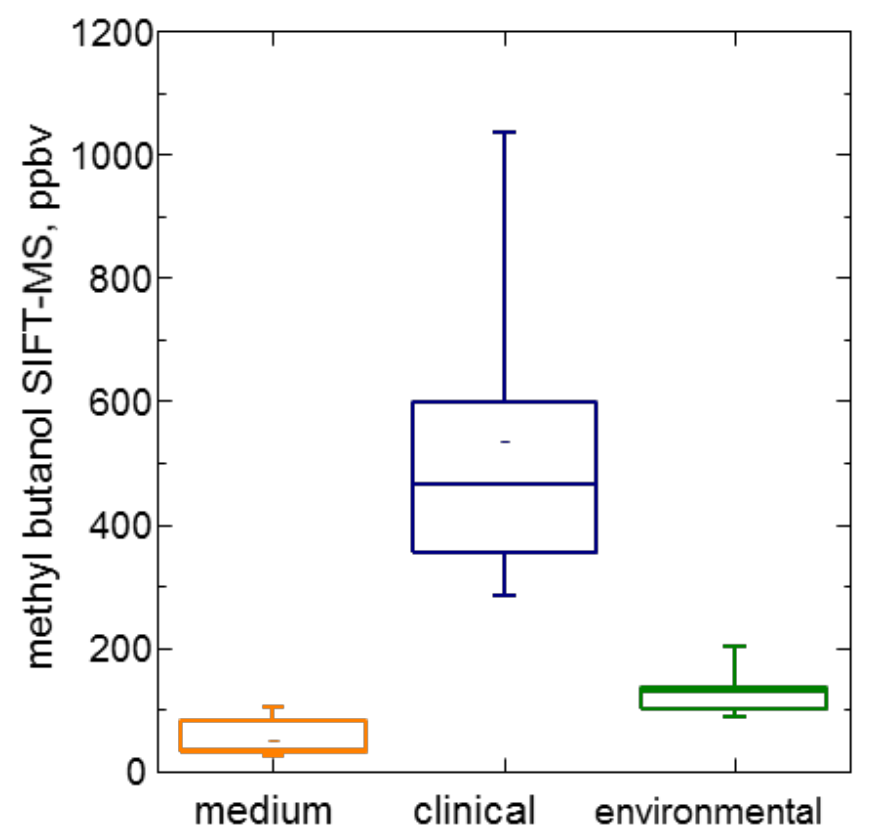

Fig. 4. Concentrations (ppbv) of total methyl butanol measured using SIFT-MS obtained for the headspace of 15 clinical and 5 environmental Stenotrophomonas strains cultured in liquid MHB medium. Also included, for comparison, are the results from identically cultivated medium samples that were not inoculated.

SIFT-MS quantification of total methyl butanol (sum of the concentrations of the two isomers) showed that these compounds were produced in the headspace of all 20 strains in significant concentrations within the range 87-1036 parts-per-billion by volume (ppbv). As shown by the box and whisker plots in Fig. 4, there is a clear difference between the concentrations of total methyl butanol in the headspace of the environmental strains (from 87 to 201ppbv) and the clinical strains (from 283 to 1036 ppbv.

Some clinical strains of S. maltophilia (genogroups other than GG9) produced much more methyl butanol than the environmental S. maltophilia (GG9) and S. rhizophila. This difference may result from the genetic distinction of the two bacterial species and different genogroups of S. maltophilia. If this is the case, such a difference could assist diagnosis of lung/airways infection by different Stenotrophomonas genogroups via breath analysis. However, more strains must be analysed using different culture media 
before any definite conclusion can be drawn on this issue. In addition, the quantitative differences in the production of methyl butanol between the two bacterial groups may also reflect the different cultivation temperatures for the clinical $\left(37^{\circ} \mathrm{C}\right)$ and environmental $\left(30^{\circ} \mathrm{C}\right)$ strains that were needed to obtain approximately equal culture densities for all strains.

It is also important to note that methyl butanol has also been identified in the headspace of cultures of the major CF pathogen P. aeruginosa when cultured in MHB liquid medium [33, 34], but at much lower concentrations (within the range $1-15$ ppbv) [13]. This may further support the role of methyl butanol as a specific biomarker of Stenotrophomonas infection. Again, this assumption needs to be verified by more extensive studies.

Only a very weak correlation $\left(\mathrm{R}^{2}=0.07\right)$ is observed between the GC-MS peak area for methyl butanol and the total concentration of methyl butanol isomers as quantified by SIFT-MS. This is most probably due to the uncertainty in defining GC-MS peak areas and the variability of the extraction efficiency of volatile metabolites by the SPME fibre. Fig. 5 shows a comparison of the total GC-MS peak areas for methyl butanol and butanol in the headspace of all 20 Stenotrophomonas strains. The reasonable correlation $\left(\mathrm{R}^{2}=0.6\right)$ may suggest common biochemical origins for these isomers (see Appendix 2). 


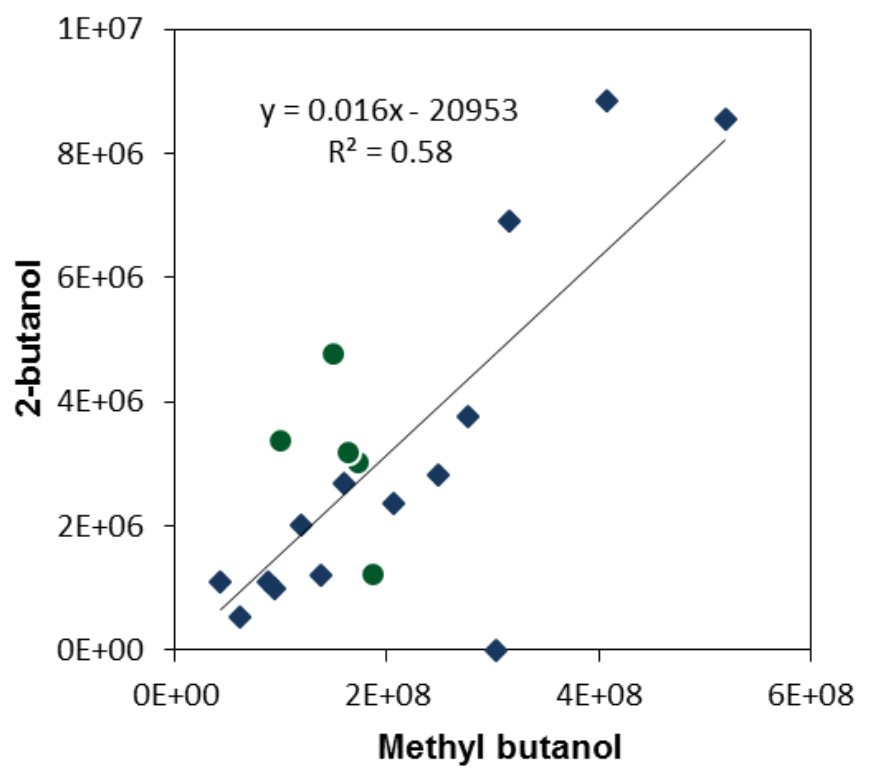

Fig. 5 GC-MS peak area correlation for total methyl butanol and 2-butanol detected in the headspace of 20 Stenotrophomonas strains cultured in liquid MHB medium. The diamonds indicate the 15 clinical strains and the circles indicate the 5 environmental strains.

\section{Principal component analysis (PCA) of SIFT-MS concentrations data}

Principal component analysis (PCA) methods, as used previously for the assessment of bacterial culture headspace data obtained using SIFT-MS [5, 35], have also been used in the present work to visualize the differences in the composition headspace concentration of VOCs emitted by the clinical and environmental Stenotrophomonas strains cultured in the same medium. The plot of the PCA scores of the first two principal components shown in Fig. 6 reveals a clear discrimination between the concentrations of volatile metabolites present in the headspace of clinical and environmental Stenotrophomonas bacterial cultures and culture medium alone. The main compounds important for discrimination between the environmental and the clinical strains are acetaldehyde, aminoacetophenone, ammonia, carbon disulphide and dimethyl disulphide, as indicated by the directions of the eigenvectors plotted in Fig. 6. 


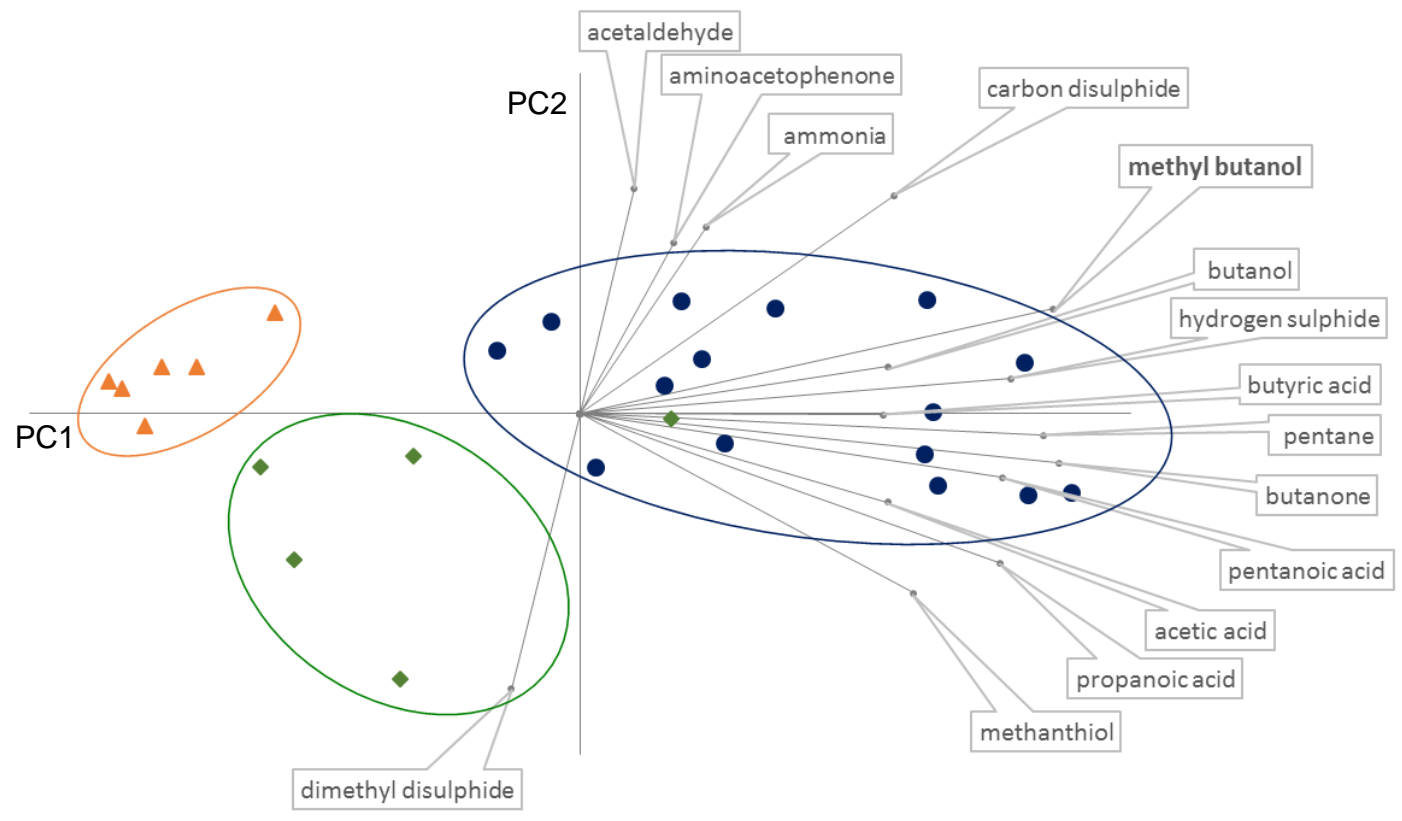

Fig 6. Principal component analysis (PCA) for SIFT-MS data obtained from full spectral scans. Scores of the first two principal components PC1 and PC2, which correspond to headspace concentrations, are indicted by blue circles for the 15 clinical strains, green diamonds for the 5 environmental strains and orange triangles for identically cultivated medium samples that were not inoculated. The lines indicate the projected directions corresponding to the individual compounds indicated. The PC1 scores plotted on the horizontal axis explain $29.5 \%$ of the variation and the PC2 scores plotted on the vertical axis explain 13\% of the total variation.

\section{Concluding remarks}

The results of this combined SPME/GC-MS and SIFT-MS study indicate that methyl butanol is produced by both environmental and clinical strains of the genus Stenotrophomonas cultured in vitro, and that clinical strains produced methyl butanol in significantly higher concentrations compared to strains from the natural environment. Thus, methyl butanol might be present in measurable concentration in the exhaled breath of CF patients; if so, this could provide a specific and valuable non-invasive VOC diagnostic of early lung infection by Stenotrophomonas. Note that methyl butanol was not reported in previous studies of pathogens relevant to CF, viz. P. aeruginosa, S. aureus, S. pneumoniae, and the fungus 
A. fumigatus [6]. More extensive work is needed to investigate this tentative postulate, including further in vitro bacterial culture studies as well as in vivo studies of the exhaled breath of precisely defined clinically and epidemiologically cohorts of patients. Also, it is important to keep in mind that the suggested method of SIFT-MS quantification of methyl butanol relies on the use of the analyte ions at $\mathrm{m} / \mathrm{z} 71,89$ and 107 formed by its reactions with the $\mathrm{H}_{3} \mathrm{O}^{+}$reagent ions. Whilst currently no overlaps with common breath metabolites are known at these $m / z$ values, this cannot be entirely ruled out.

This study again shows that by direct analysis of the headspace of active bacterial cultures by SIFT-MS, absolute concentrations of several volatile metabolites can be obtained simultaneously. Reporting of absolute concentrations is most valuable in such in vitro experiments when the ultimate objective of the work is to provide guidance to envisaged in vivo investigations and the anticipated exploitation of breath analysis for the detection bacterial infection in the airways and lungs. Diagnosis of airways infection by sample (sputum, lavage) collection is often challenging, especially in children, and so immediate non-invasive diagnosis by the identification and quantification in exhaled breath of volatile biomarkers of specific infections is very attractive. Such could provide a rapid identification of the infecting bacterium, thus indicating more appropriate treatment, and a concomitant reduction of stress for both patients and clinicians alike.

\section{Acknowledgements}

We gratefully acknowledge funding from Czech Science Foundation GACR projects No 14-14534S and No 14-15771P. We are grateful to Martina Maixnerová for help with culturing of bacterial strains. 
Appendix 1 Genetic diversity of the genus Stenotrophomonas

The genus Stenotrophomonas currently comprises 12 distinct species with valid names (http://www.bacterio.net/stenotrophomonas.html), but the genetic diversity is remarkable and further differentiation at the species level is expected [36]. This applies also to the most important species, Stenotrophomonas maltophilia, which has been only broadly defined and may represent several species according to the current taxonomic criteria. A number of genotypic methods have been used to unravel the taxonomic and phylogenetic diversity of S. maltophilia, including AFLP fingerprinting [37], 16S rRNA gene comparative analysis [20] and multilocus sequence typing (MLST) [17]. Most strains of $S$. maltophilia are characterized by their resistance to many currently available broad-spectrum antimicrobial agents [38]. The environmental strains of S. maltophilia show high resistance to antibiotics, as do the strains isolated from the sputum of CF patients, and the resistance profile pattern of the strains is seen to be independent of their source of isolation [39]. Many strains are susceptible to co-trimoxazole and ticarcillin, though resistance to these antibiotics has been increasing [40].

Strains from AFLP group 8 (= MLST genogroup (GG) 8 = 16S rRNA group E1) were recently accommodated in a novel species, Stenotrophomonas rhizophila [19]. This species has been isolated from environmental sites only. Another distinct lineage, AFLP group 9 (MLST genogroup GG9 = 16S rRNA group E2), also seems to be a strictly environmental group [18-20]. In contrast, the AFLP group 6 (= MLST genogroup GG6) has repeatedly been shown to be prominent among clinical strains [16, 17, 37]. The defining characteristics of S. rhizophila are as follows: growth at $4{ }^{\circ} \mathrm{C}$ and the absence of growth at $40{ }^{\circ} \mathrm{C}$; the utilization of xylose as a carbon source; lower osmotic tolerance, the absence of lipase and $\beta$ glucosidase production and antifungal activity [41]. It has been observed that S. rhizophila, as a plantassociated bacterium, has the ability to grow at lower temperatures. As the environmental strains have a preference for lower temperatures, this might be related to their ability to survive in the rhizosphere. 
Appendix 2 Biochemical routes to the production of methyl butanol by the genus Stenotrophomonas

There are several known mechanisms of production of butanol and methyl butanol by bacteria. For example, butanol (biobutanol) can be naturally synthesized by solventogenic bacteria of the genus Clostridium through renewable substrates such as glucose. Higher-order alcohols (like 3-methyl butanol) can be produced through the ketoacid pathways [42, 43]. Scheme I describes possible and natural fermentative pathways for biosynthesis of branched-chain higher alcohols, including methyl butanol. This shows that metabolic synthesis of both methyl butanol and butanol is complementary. It is perhaps significant that both of these VOCs have been identified in measurable concentration in the headspace of the cultures of all 20 strains included in the present study, and that their headspace concentrations are well correlated, as seen in Fig. 5, probably indicating that they both originate from the keto-acid metabolic pathway (see Scheme I). Much previous research has been concerned with the biotechnology of methyl butanol production as a potential biofuel and so some details of the mechanism of methyl butanol production by different bacteria and yeast cells are already understood. A high yield for iso-butanol production from glucose by microbial fermentation has been achieved [44].

Scheme I shows that the two isomers of methyl butanol each originate from a different metabolic pathway: 3-methyl butanol from that proceeding via pyruvate and 2 methyl butanol from that proceeding via oxaloacetate, whilst butanol is produced in both of these pathways. The clinical and environmental strains included in the present study are genetically different and thus could produce different amount of enzymes. 


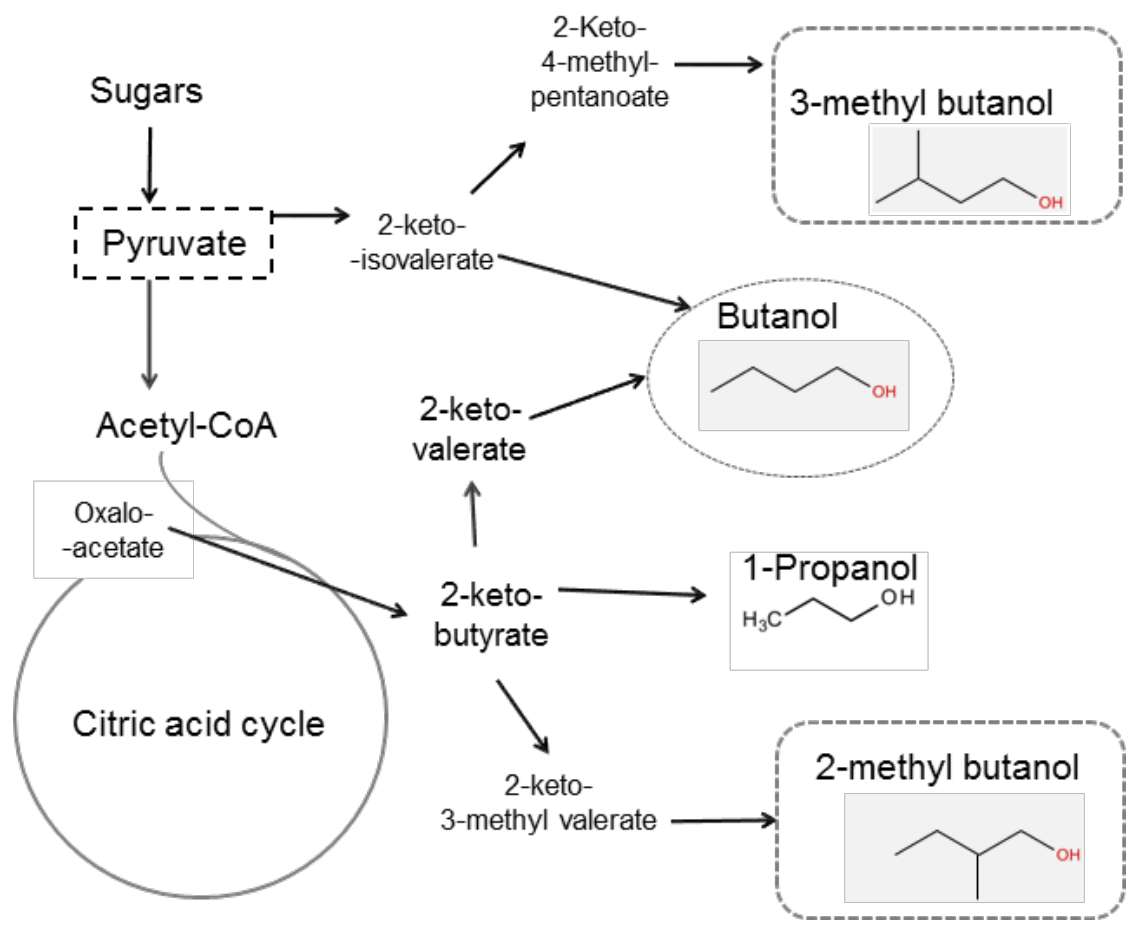

Scheme I Metabolic keto-acid pathway for the biosynthetic production of 3-methyl butanol and 2-methyl butanol $[42,44]$

Another possible synthesis of methyl butanol is from methyl butanal, even though is not considered to be physiologically relevant:

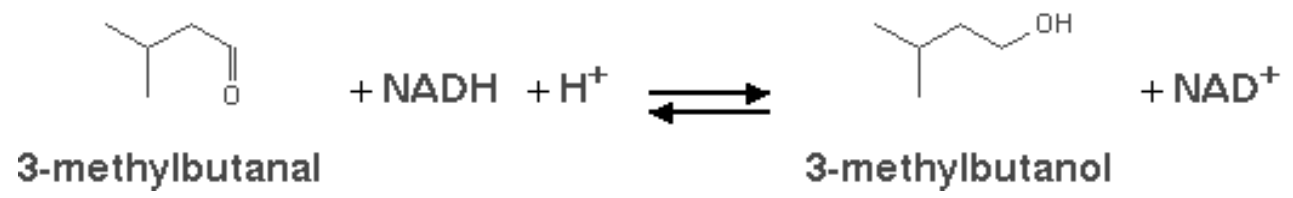

\section{3-methylbutanal}

3-methylbutanol

\section{Scheme II}

However, the correlation between methyl butanol and methyl butanal concentrations as measured in the present study of Stenotrophomonas is weak $\left(\mathrm{R}^{2}=0.16\right)$. Thus, we judge that this metabolic pathway for methyl butanol production by this bacterium is unlikely. 
S. maltophilia and S. rhizophila are ubiquitous in aqueous environments and soil. As organisms living in a wild environment, Stenotrophomonas have to coexist with many other bacteria and fungi that can produce toxic secondary metabolites. Thus, the competition between microorganisms and Stenotrophomonas bacteria for these ecological niches is very intense. Stenotrophomonas is also often associated with plants and has been isolated from rhizosphere in which wheat, oat, cucumber, maize, oilseed rape, and potato are grown. Consequently, Stenotrophomonas bacteria must have some specific strategies to exist in such environments and to manage the symbiotic relationship [45]. It is often a dominant member of the rhizosphere microbial community of plants and can produce high amounts of the plant growth hormone indole-3-acetic acid [39]. On the other hand, some studies suggest that Stenotrophomonas might potentially produce phytotoxic substance at one of stages of its life cycle, [46] causing root shortening due to 2-methyl butanol, 3-methyl butanol and 3-methyl butanal. Thus, we speculate that the high production of methyl butanol by Stenotrophomonas could be a specific geneticallyconditioned pathway process in Stenotrophomonas bacteria.

From the evolutionary point-of-view, it is worth noting that methyl butanol production was detected in both the clinical and environmental Stenotrophomonas strains. Many studies have consistently shown that the environmental genogroups GG9 (syn. E2) and GG8 (S. rhizophila, syn. E1) branch at the base of Stenotrophomonas phylogenetic trees, and can therefore be considered ancestral lineages [18-20]. Thus, it can be inferred that the metabolic pathways leading to methyl butanol production were fixed early during Stenotrophomonas evolution before the divergence of pathogenic lineages (typically GG6). However, cellular metabolism of Stenotrophomonas is very complex and it is therefore difficult to account for every detail of the synthesis of methyl butanol by this bacterium. For effective optimization, details on the genes and enzymes responsible for the synthesis of the target compound, as well as undesired side products, need to be known. 


\section{References}

[1] Berg G, Eberl L and Hartmann A 2005 The rhizosphere as a reservoir for opportunistic human pathogenic bacteria Environmental Microbiology 7 1673-85

[2] Yong Y C and Zhong J J 2010 Recent advances in biodegradation in China: New microorganisms and pathways, biodegradation engineering, and bioenergy from pollutant biodegradation Process Biochemistry 45 1937-43

[3] Brooke J S 2012 Stenotrophomonas maltophilia: an Emerging Global Opportunistic Pathogen Clinical Microbiology Reviews 25 2-41

[4] Smith D, Španěl P, Gilchrist F J and Lenney W 2013 Hydrogen cyanide, a volatile biomarker of Pseudomonas aeruginosa infection J. Breath Res. 7044001

[5] Chippendale T W E, Gilchrist F J, Španěl P, Alcock A, Lenney W and Smith D 2014 Quantification by SIFT-MS of volatile compounds emitted by in vitro cultures of S. aureus, S. pneumoniae and $\mathrm{H}$. influenzae isolated from patients with respiratory diseases Analytical Methods 6 2460-72

[6] Chippendale T W E, Gilchrist F J, Španěl P, Alcock A, Lenney W and Smith D 2014 Quantification by SIFT-MS of volatile compounds emitted by Aspergillus fumigatus cultures and in coculture with Pseudomonas aeruginosa, Staphylococcus aureus and Streptococcus pneumoniae Analytical Methods 6 8154-64

[7] Waters V 2012 New Treatments for Emerging Cystic Fibrosis Pathogens other than Pseudomonas Current Pharmaceutical Design 18 696-725

[8] Julak J, Stranska E, Rosova V, Geppert H, Španěl P and Smith D 2006 Bronchoalveolar lavage examined by solid phase microextraction, gas chromatography-mass spectrometry and selected ion flow tube mass spectrometry J. Microbiol. Methods 65 76-86

[9] Enderby B, Smith D, Carroll W and Lenney W 2009 Hydrogen Cyanide as a Biomarker for Pseudomonas Aeruginosa in the Breath of Children With Cystic Fibrosis Pediatr. Pulmonol. 44 142-7

[10] Smith D and Španěl P 2005 Selected ion flow tube mass spectrometry (SIFT-MS) for on-line trace gas analysis Mass Spectrom. Rev. 24 661-700

[11] Španěl P and Smith D 2011 Progress in SIFT-MS; breath analysis and other applications Mass Spectrom. Rev. 30 236-67

[12] Shestivska V, Nemec A, Drevinek P, Sovová K, Dryahina K and Španěl P 2011 Quantification of methyl thiocyanate in the headspace of Pseudomonas aeruginosa cultures and in the breath of cystic fibrosis patients by selected ion flow tube mass spectrometry Rapid Commun. Mass Spectrom. 25 245967

[13] Shestivska V, Španěl P, Dryahina K, Sovová K, Smith D, Musilek M and Nemec A 2012 Variability in the concentrations of volatile metabolites emitted by genotypically different strains of Pseudomonas aeruginosa J. Appl. Microbiol. 113 701-13

[14] Filipiak W, Sponring A, Baur M M, Filipiak A, Ager C, Wiesenhofer H, Nagl M, Troppmair J and Amann A 2012 Molecular analysis of volatile metabolites released specifically by staphylococcus aureus and pseudomonas aeruginosa Bmc Microbiology 1216

[15] Bean H D, Dimandja J M D and Hill J E 2012 Bacterial volatile discovery using solid phase microextraction and comprehensive two-dimensional gas chromatography-time-of-flight mass spectrometry Journal of Chromatography B-Analytical Technologies in the Biomedical and Life Sciences 901 41-6

[16] Nunvar J, Drevinek P and Licha I 2012 DNA profiling of Stenotrophomonas maltophilia by PCR targeted to its species-specific repetitive palindromic sequences Letters in Applied Microbiology 54 5966 
[17] Kaiser S, Biehler K and Jonas D 2009 A Stenotrophomonas maltophilia Multilocus Sequence Typing Scheme for Inferring Population Structure Journal of Bacteriology 191 2934-43

[18] Adamek M, Overhage J, Bathe S, Winter J, Fischer R and Schwartz T 2011 Genotyping of Environmental and Clinical Stenotrophomonas maltophilia Isolates and their Pathogenic Potential Plos One 6 e27615

[19] Vasileuskaya-Schulz Z, Kaiser S, Maier T, Kostrzewa M and Jonas D 2011 Delineation of Stenotrophomonas spp. by multi-locus sequence analysis and MALDI-TOF mass spectrometry Systematic and Applied Microbiology 34 35-9

[20] Minkwitz A and Berg G 2001 Comparison of antifungal activities and 16S ribosomal DNA sequences of clinical and environmental isolates of Stenotrophomonas maltophilia Journal of Clinical Microbiology 39 139-45

[21] Stein S E 2010 Mass Spectra. In: Linstrom PJ, Mallard WG, eds. NIST Chemistry WebBook, NIST Standard Reference Database Number 69 Gaithersburg: National Institute of Standards and Technology) p.

[22] Allardyce R A, Langford V S, Hill A L and Murdoch D R 2006 Detection of volatile metabolites produced by bacterial growth in blood culture media by selected ion flow tube mass spectrometry (SIFTMS) J. Microbiol. Methods 65 361-5

[23] Španěl P, Dryahina K and Smith D 2006 A general method for the calculation of absolute trace gas concentrations in air and breath from selected ion flow tube mass spectrometry data Int. J. Mass Spectrom. 249 230-9

[24] Dryahina K and Španěl P 2005 A convenient method for calculation of ionic diffusion coefficients for accurate selected ion flow tube mass spectrometry, SIFT-MS Int. J. Mass Spectrom. 244 148-54

[25] Španěl P and Smith D 2013 Advances in On-line Absolute Trace Gas Analysis by SIFT-MS Current Analytical Chemistry 9 525-39

[26] Bos L D J, Sterk P J and Schultz M J 2013 Volatile Metabolites of Pathogens: A Systematic Review PLOS Path. 9

[27] Greenman J, Saad S, Hewett K, Thorn R M S and Reynolds D M 2013 Review: In vitro biofilm models for studying oral malodour Flavour and Fragrance Journal 28 212-22

[28] Ross B M 2011 Changes in oral trace gas concentrations following orthognathic surgery and intermaxillary fixation: a case study using selected ion flow tube mass spectrometry International Journal of Oral Science 3 160-4

[29] Lux M F, Keith E, Hsu T and Drake H L 1990 Biotransformations of Aromatic-Aldehydes by Acetogenic Bacteria Fems Microbiology Letters 67 73-7

[30] Tidswell E C and Morris J G 1993 Anaerobic Bacterial Reductions of Aldehydes and Ketones - a Rapid Screening-Procedure Letters in Applied Microbiology 17 242-7

[31] Sule-Suso J, Pysanenko A, Španěl P and Smith D 2009 Quantification of acetaldehyde and carbon dioxide in the headspace of malignant and non-malignant lung cells in vitro by SIFT-MS Analyst 134 2419-25

[32] Filipiak W, Sponring A, Mikoviny T, Ager C, Schubert J, Miekisch W, Amann A and Troppmair J 2008 Release of volatile organic compounds (VOCs) from the lung cancer cell line CALU-1 in vitro Cancer Cell International 817

[33] Labows J N, McGinley K J, Webster G F and Leyden J J 1980 Headspace analysis of volatile metabolites of Pseudomonas-aeruginosa and related species by gass chromatography-mass spectrometry Journal of Clinical Microbiology 12 521-6 
[34] Savelev S U, Perry J D, Bourke S J, Jary H, Taylor R, Fisher A J, Corris P A, Petrie M and De Soyza A 2011 Volatile biomarkers of Pseudomonas aeruginosa in cystic fibrosis and noncystic fibrosis bronchiectasis Letters in Applied Microbiology 52 610-3

[35] Sovova K, Cepl J, Markos A and Španěl P 2013 Real time monitoring of population dynamics in concurrent bacterial growth using SIFT-MS quantification of volatile metabolites Analyst 138 4795-801

[36] Ryan R P, Monchy S, Cardinale M, Taghavi S, Crossman L, Avison M B, Berg G, van der Lelie D and Dow J M 2009 The versatility and adaptation of bacteria from the genus Stenotrophomonas Nature Reviews Microbiology 7 514-25

[37] Hauben L, Vauterin L, Moore E R B, Hoste B and Swings J 1999 Genomic diversity of the genus Stenotrophomonas International Journal of Systematic Bacteriology 49 1749-60

[38] Denton M and Kerr K G 1998 Microbiological and clinical aspects of infection associated with Stenotrophomonas maltophilia Clinical Microbiology Reviews 11 57-+

[39] Berg G, Roskot N and Smalla K 1999 Genotypic and phenotypic relationships between clinical and environmental isolates of Stenotrophomas maltophilia Journal of Clinical Microbiology 37 3594-600 [40] Tsiodras S, Pittet D, Carmeli Y, Eliopoulos G, Boucher H and Harbarth S 2000 Clinical implications of Stenotrophomonas maltophilia resistant to trimethoprim-sulfamethoxazole: a study of 69 patients at 2 university hospitals Scandinavian Journal of Infectious Diseases 32 651-6

[41] Wolf A, Fritze A, Hagemann M and Berg G 2002 Stenotrophomonas rhizophila sp nov., a novel plant-associated bacterium with antifungal properties International Journal of Systematic and Evolutionary Microbiology 52 1937-44

[42] Connor M R, Cann A F and Liao J C 2010 3-Methyl-1-butanol production in Escherichia coli: random mutagenesis and two-phase fermentation Appl. Microbiol. Biotechnol. 86 1155-64

[43] Shen C R and Liao J C 2008 Metabolic engineering of Escherichia coli for 1-butanol and 1propanol production via the keto-acid pathways Metabolic Engineering 10 312-20

[44] Atsumi S, Hanai T and Liao J C 2008 Non-fermentative pathways for synthesis of branched-chain higher alcohols as biofuels Nature 451 86-U13

[45] Juhnke M E, Mathre D E and Sands D C 1989 Relationship between bacterial seed inoculum density and rhizosphere colonization of spring wheat Soil Biology \& Biochemistry 21 591-5

[46] Pacioni G 1991 Effects of tuber metabolites on the rhizospheric environment Mycological Research 95 1355-8 